\title{
Fly-casting in protein-DNA binding: Frustration between protein folding and electrostatics facilitates target recognition
}

\author{
Yaakov Levy, Jose N. Onuchic, and Peter G. Wolynes
}

\section{Supporting information}

The dynamic nature of the association of the SAP-1 protein to its specific DNA binding site was studied using a native topology based model (Gō model) supplemented by non-specific electrostatic interactions. The native topology based model takes into account only interactions that exist in the native structure and, therefore, includes mainly topological frustration. Adding nonspecific electrostatic interactions contribute energetic frustration between the protein and the DNA as well as within the protein. Pure Gō-model has already been used to study the folding of many monomeric proteins that fold in a two-state fashion ${ }^{1-5}$ or a more complicated folding scheme as well as to study higher molecularity reactions such as dimerization and tetramerization $^{6-9}$. A significant correlation is found between the experimental folding rates and the rates (or the free energy barrier heights) obtained from the topology based simulations. In addition, the Gō model was successfully used to predict intermediates observed experimentally during the folding of larger proteins. The impressive agreement between the results for perfectly funneled energy landscapes, and experimental studies strongly indicates the validity of the idea that proteins are energetically minimally frustrated for both folding and binding.

In our study the protein and the DNA are modeled using a reduced representation. Each residue is represented by a single bead centered on its $\alpha$-carbon $\left(\mathrm{C}_{\alpha}\right)$ position. Adjacent beads are strung together into a polymer chain by means of a potential encoding bond length and angle constraints. The secondary structure is encoded in the dihedral angle potential and the nonbonded (native contact) potential. In the framework of the model, all native contacts are represented by the 10-12 Lennard Jones form without any discrimination between the various chemical types of interaction. The residues do not have any chemical identity (the information for folding is encoded in the structure) but some have a point charge. Accordingly, positively charged residues (Arg and Lysine) have positive point charge and the negatively charged residue (Asp and Glu) have a negative point charge. The other beads are neutral. The interaction energy $U$ at a given protein conformation $\Gamma$ is given by 


$$
\begin{aligned}
U\left(\Gamma, \Gamma_{0}\right)= & \sum_{\text {bonds }}^{N-1} K_{b}\left(b_{i}-b_{0 i}\right)^{2}+\sum_{\text {angles }}^{N-2} K_{\theta}\left(\theta_{i}-\theta_{0 i}\right)^{2} \\
& +\sum_{\text {dihedrals }}^{N-3}\left\{K_{\phi}^{(1)}\left[1-\cos \left(n \times\left(\phi_{i}-\phi_{0 i}\right)\right)\right]+K_{\phi}^{(3)}\left[1-\cos \left(n \times\left(\phi_{i}-\phi_{0 i}\right)\right)\right]\right. \\
& \sum_{i>j} \frac{332 q_{i} q_{j}}{\mathcal{E}_{\text {dielc }} r_{i j}^{2}}+\sum_{\begin{array}{c}
\text { native-contacts } \\
|i-j|>3
\end{array}}\left\{\varepsilon\left[5\left(\frac{r_{0 i j}}{r_{i j}}\right)^{12}-6\left(\frac{r_{0 i j}}{r_{i j}}\right)^{10}\right]\right\}+\sum_{\substack{\text { non-native } \\
\text { contacts }, i-j \mid>3}}\left(\frac{C}{r_{i j}}\right)^{12}
\end{aligned}
$$

In the equation, $b_{i}, \theta_{i}$, and $\phi_{i}$ stand for the $i$ th virtual bond length between $i$ th and $(i+1)$ th residue, the virtual bond angle between $(i-1)$ th and $i$ th bonds, and the virtual dihedral angle around the $i$ th bond, respectively. The parameters $b_{0 i}, \theta_{0 i}$, and $\phi_{0 i}$ stand for the corresponding variables at the native structure. $q_{i}$ and $q_{j}$ equal $1(-1)$ for positively (negatively) charged residues, or 0 for neutral residues. The strength of the coulomb interactions is controlled by the dielectric constant, $\varepsilon_{\text {dielc }}$ (Figure 1). In the current study, $\varepsilon_{\text {dielc }}$ gets values between $20-\infty$, where $\infty$ represents turning off the long-range electrostatic interactions.

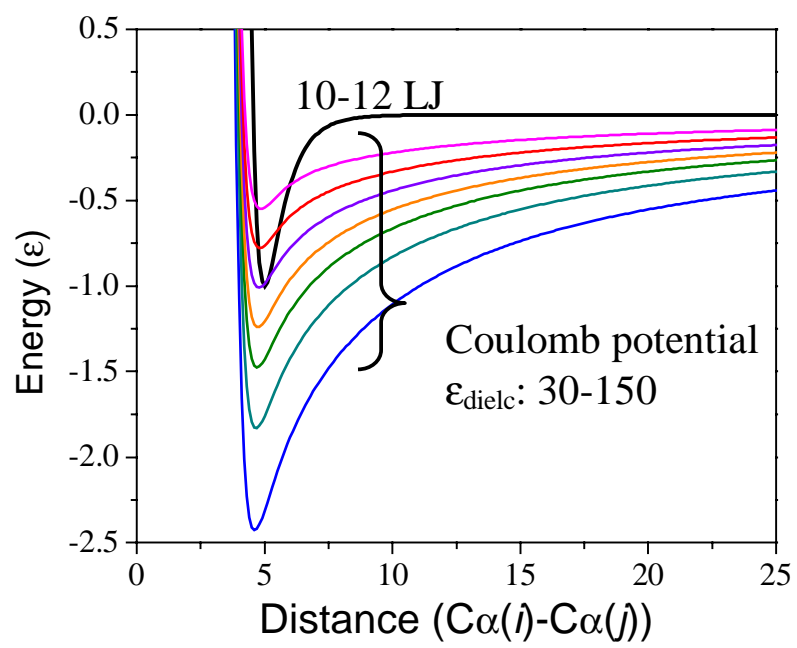

Figure 1. The energetic contributions of the short range $\mathrm{LJ}$ and the long range Coulomb interactions

In the framework of the model, all native contacts (as defined by the CSU software) are represented by the 10-12 Lennard Jones form without any discrimination between the various chemical types of interaction. Moreover, both the intra- and intermonomeric contacts (interfacial contacts) are treated in the same way without any bias toward separate folding or toward binding. 
The $r_{i j}$ and $r_{0 i j}$ are the $\mathrm{C}_{\alpha}-\mathrm{C}_{\alpha}$ distances between the contacting residues $i$ and $j$ in conformation $\Gamma$ and $\Gamma_{0}$ (the PDB structure), respectively. In the summation over nonnative contacts, $C(=4.0 \AA)$ parameterizes the excluded volume repulsion between residues pairs that do not belong to the given native contact set. In the paper, all temperatures and energies are reported in units of $\tilde{\varepsilon}$ For other parameters, we use similar values that have been used in several folding studies ${ }^{1,10,11}$, namely, $K_{b}=100.0, K_{\theta}=20.0, K_{\phi}^{(1)}=1.0, K_{\phi}^{(3)}=0.5, \varepsilon=1.0$. When a native contact is a saltbridge, its $\varepsilon$ equals 0.1 so that its total energetic contribution will be similar to other native contacts. Doing so we examine the long-range effect of charged-charged interactions rather than their energetic contributions.

To enhance the sampling of binding events, a constraint is applied on the protein-DNA system where they are confined in a sphere of radius 40 centered at the center of mass of the DNA that is kept frozen. For each electrostatic strength, several constant temperature molecular dynamics simulations were performed (using the simulation package AMBER6 as an integrator ${ }^{12}$ ) starting from an unbound (folded or unfolded) protein conformation. At a given temperature several trajectories were collected starting from different initial conformation and velocities. The length of the simulations was determined by the demand of having several transitions of folding/unfolding or binding/unbinding at the transition temperatures. The multiple trajectories were combined using the Weighted Histogram Analysis Method (WHAM) to provide the transition temperatures from the peaks of the specific heat versus temperature and to calculate thermodynamic properties of the systems. Figure 2 shows the thermodynamics features of the folding of SAP-1 protein in its free form (Fig. 2A) and in the vicinity of a DNA molecule (Fig. 2B).

A
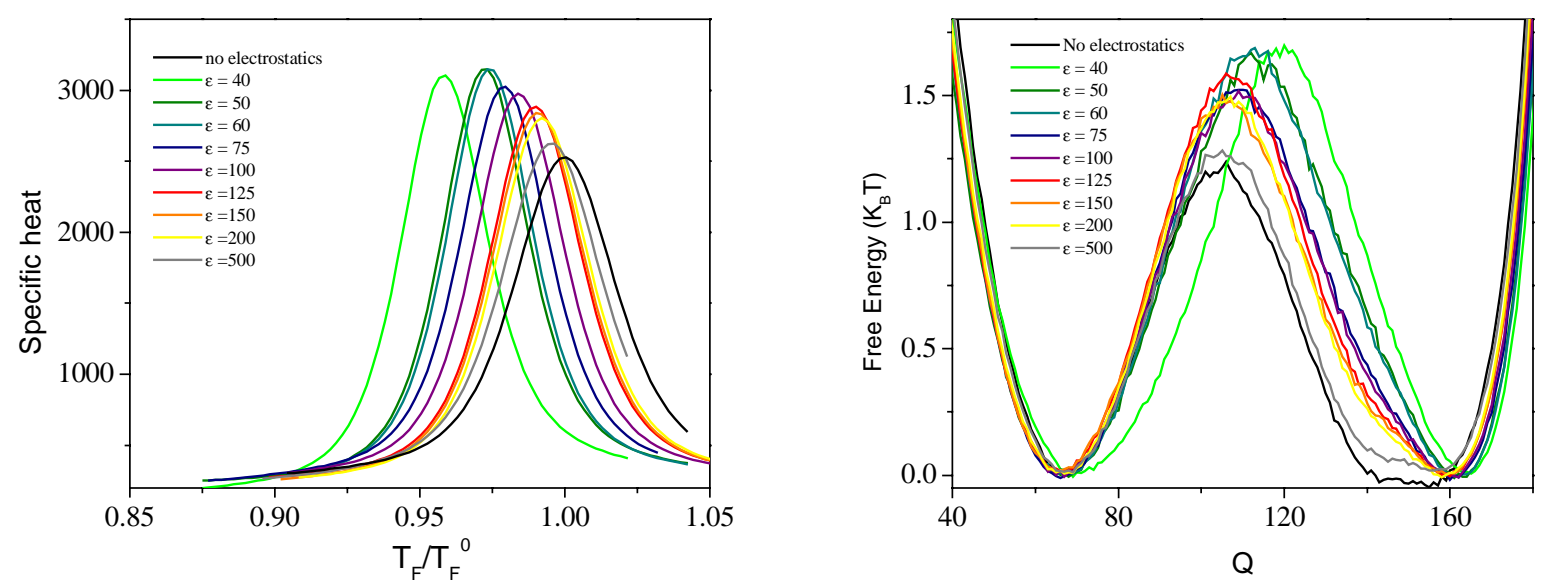

Stability and folding barrier of free SAP-1 protein. 

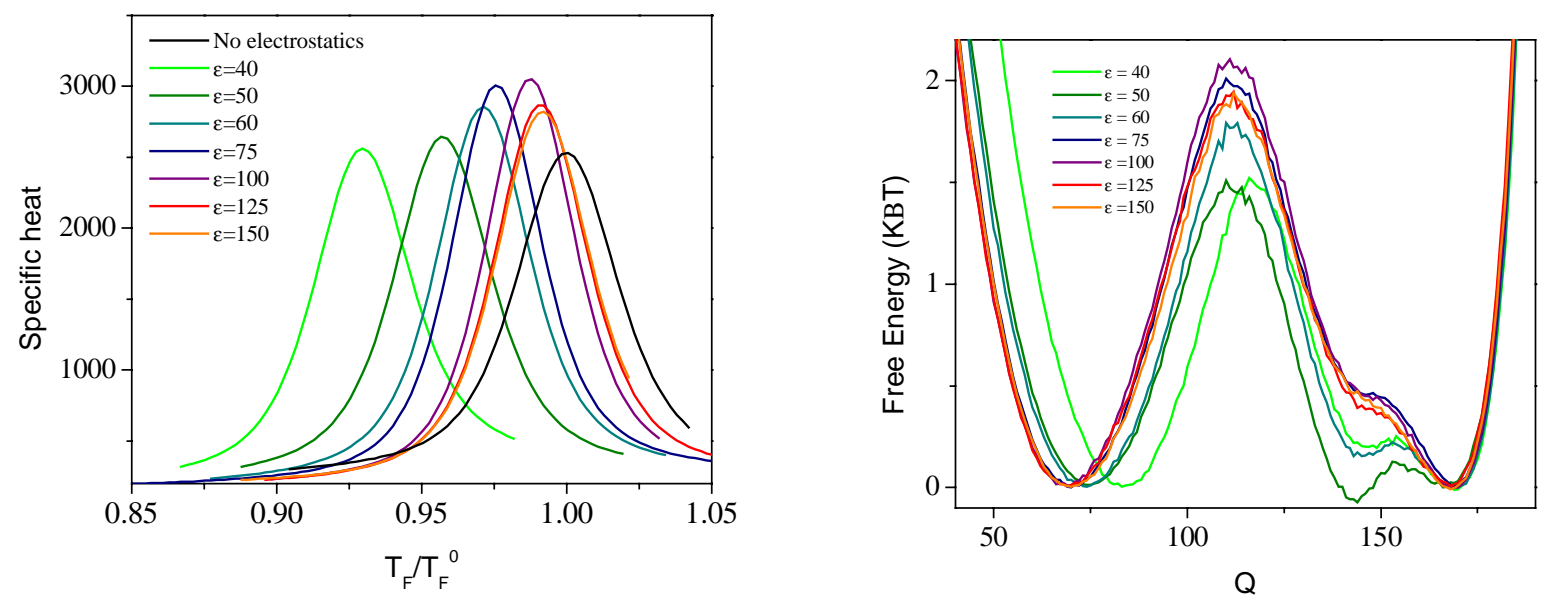

Stability and folding barrier of SAP-1 protein in the vicinity of DNA molecule

Figure 2.

Additionally, we studied the system with a model that neglects the charged-charged interactions within the protein and, accordingly, the electrostatics forces are only from proteinDNA interactions (Figure 3). We simulated this model when all electrostatic interactions at the interface are included or that the repulsive interactions were omitted. These models, while are not realistic, enabled us to estimate the effect of electrostatic forces in protein-DNA complexes on their binding mechanism. The various models suggest that while the residue-residue electrostatic forces have some effects, the principles of the mechanism of protein-DNA assembly are very similar in all the models and call for fly-casting assisted by the long-range electrostatic field (figure 4).

To estimate the specific binding affinity we simulated the SAP-1 DNA complex using two models. In the first model, the interface was represented by the specific short-range interactions (modeled as LJ) as defined by the crystal structure as well as non-specific long-range electrostatic interactions (modeled as Coulomb interactions). In the second model, the interface was modeled by the non-specific long-range electrostatic interactions only. The difference of the free energy in the bound state may represent the specific binding affinity (Figure 4C). 


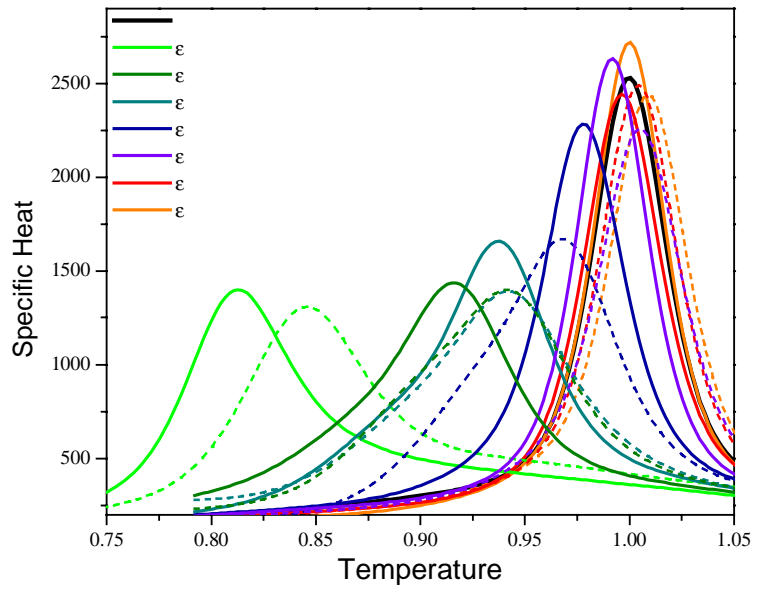

Figure 3. Cv plots of the SAP-1-DNA system for a model where residue-residue electrostatic interactions are not included.

A

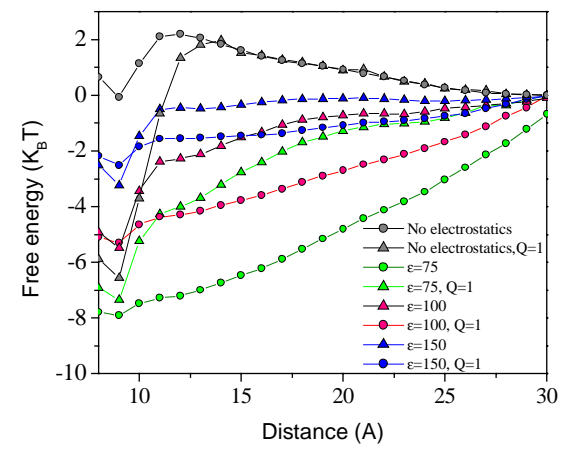

B

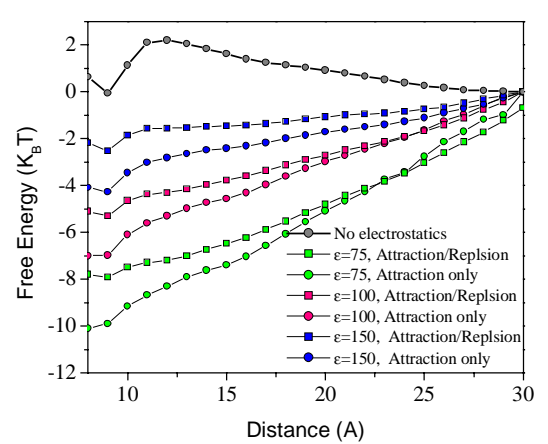

C

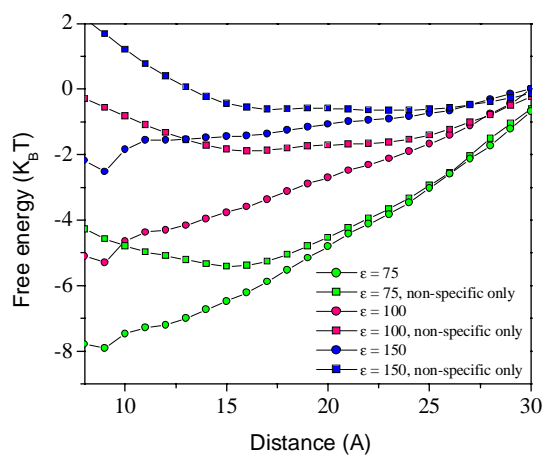

Figure 4. Fly-casting in SAP-1 binding to its specific DNA binding site. In these calculations the electrostatic interactions are only between the DNA and the protein. (A) The effect of the protein rigidity on the binding mechanism (Q equals 1 corresponds to constraining the protein to be fully folded. (B) The effect of the repulsive protein-DNA interactions (due to negatively charged residues) on the binding efficiency. (C) Comparison between specific and non-specific protein-DNA binding.

The degree of fly-casting is expected to be affected by the folding stability and kinetics. To examine the interplay between folding barrier and fly-casting effect the folding barrier was tuned by gradually adding cooperativity to its folding (Figure 5). 


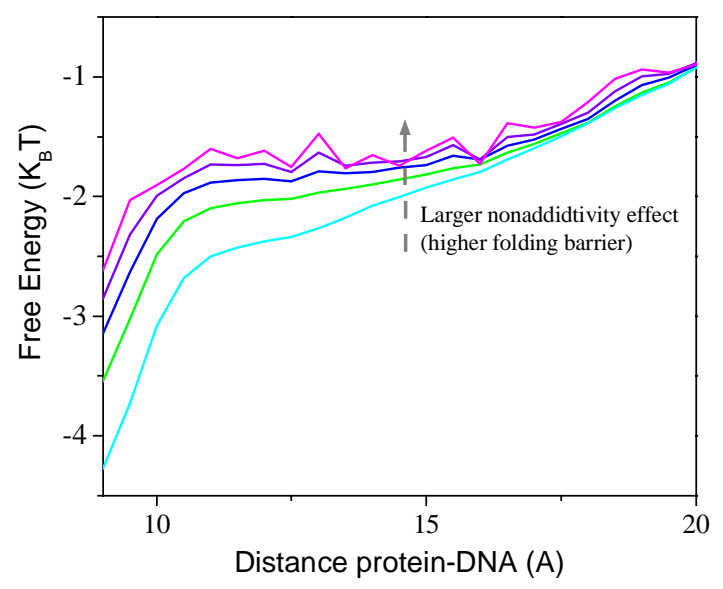

Figure 5. The effect of folding barrier on fly-casting binding kinetics

1. Clementi, C.; Nymeyer, H.; Onuchic, J. N., Toplogical and energetical factors: What determines the structural details of the transition state ensemble and "En-route" intermediate for protein folding? An investigation of small globular proteins. Journal of Molecular Biology 2000, 298, 937-953.

2. Chavez, L. L.; Onuchic, J. N.; Clementi, C., Quantifying the roughness on the Free Energy Landscape: Entropic bottlenecks and protein folding rates. J Am Chem Soc 2004, 126, (27), 84268432.

3. Clementi, C.; Garcia, A. E.; Onuchic, J. N., Interplay among tertiary contacts, secondary structure formation and side-chain packing in the protein folding mechanism all-atom representation study of protein L. J Mol Biol 2003, 326, (3), 879-890.

4. Clementi, C.; Jennings, P. A.; Onuchic, J. N., How native-state topology affects the folding of dihydrofolate reductase and interleukin-1 b. Proc Natl Acad Sci USA 2000, 97, 5871-5876.

5. Finke, J. M.; Onuchic, J. N., Equilibrium and kinetic folding pathways of a TIM barrel with a funneled energy landscape. Biophysical Journal 2005, 89, (1), 488-505.

6. Levy, Y.; Caflisch, A.; Onuchic, J. N.; Wolynes, P. G., The folding and dimerization of HIV-1 protease: evidence for a stable monomer from simulations. J Mol Biol 2004, 340, 67-79.

7. Levy, Y.; Cho, S. S.; Onuchic, J. N.; Wolynes, P. G., A survey of flexible protein binding mechanisms and their transition states using native topology based energy landscapes. $\mathrm{J} \mathrm{Mol} \mathrm{Biol}$ 2005, 346, 1121-1145.

8. Levy, Y.; Onuchic, J., Mechanisms of protein assembly: lessons from minimalist models. Acc Chem Res 2006, 39, 284-290.

9. Levy, Y.; Wolynes, P. G.; Onuchic, J. N., Protein topology determines binding mechanism. Proceedings of the National Academy of Sciences of the United States of America 2004, 101, (2), 511516.

10. Koga, N.; Takada, S., Roles of native topology and chain-length scaling in protein folding: a simulation study with a Go-like model. Journal of Molecular Biology 2001, 313, 171-180.

11. Kaya, H.; Chan, H. S., Solvation effects and driving forces for protein thermodynamic and kinetic cooperativity: how adequate is native-centric topological modeling? J Mol Biol 2003, 326, 911-931.

12. Ferrenberg, A. M.; Swendsen, R. H., Optimized Monte Carlo data analysis. Phys Rev Lett 1989, 63, 1195-1198. 